\title{
An interactive decision support system for an aggregate production planning model based on multiple criteria mixed integer linear programming
}

\author{
Carlos Gomes da Silva ${ }^{\mathrm{a}}$, José Figueira ${ }^{\mathrm{b}, \mathrm{c}}$, João Lisboa ${ }^{\mathrm{b}, \mathrm{d}, *}$, Samir Barman ${ }^{\mathrm{e}}$ \\ ${ }^{a}$ Leiria Polytechnic School of Technology and Management, Alto do Vieiro, Morro do Lena, 2401-951 Leiria, Portugal \\ ${ }^{\mathrm{b}}$ School of Economics, University of Coimbra, Av. Dias da Silva, 165, 3004-512 Coimbra, Portugal \\ ${ }^{\mathrm{c}}$ LAMSADE, University of Paris-Dauphine, Place du Maréchal De Lattre de Tassigny, 75775 Paris, Cedex 16, France \\ ${ }^{\mathrm{d}}$ Instituto de Sistemas e Robótica, University of Coimbra, Pinhal de Marrocos, Polo II, Portugal \\ e Price College of Business, University of Oklahoma, 307 W. Brooks, Norman, OK 73019, USA
}

Received 25 July 2002; accepted 24 August 2004

\begin{abstract}
In this paper, we present an aggregate production planning (APP) model applied to a Portuguese firm that produces construction materials. A multiple criteria mixed integer linear programming (MCMILP) model is developed with the following performance criteria: (1) maximize profit, (2) minimize late orders, and (3) minimize work force level changes. It includes certain operational features such as partial inflexibility of the work force, legal restrictions on workload, work force size (workers to be hired and downsized), workers in training, and production and inventory capacity. The purpose is to determine the number of workers for each worker type, the number of overtime hours, the inventory level for each product category, and the level of subcontracting in order to meet the forecasted demand for a planning period of 12 months. Additionally, a decision support system (DSS) based on the MCMILP model is proposed. It will help practitioners find the "best" solution for an APP problem without having to familiarize themselves with the mathematical complexities associated with the model. An example to illustrate the use of the DSS is also included.
\end{abstract}

(C) 2004 Elsevier Ltd. All rights reserved.

Keywords: Production management; Aggregate planning; Multiple criteria model; Interactive approach; Decision support systems

\section{Introduction}

The aggregate production planning (APP) problem is about determining the optimum production, work force, and inventory levels for each period of the planning

\footnotetext{
* Corresponding author. School of Economics, University of Coimbra, Av. Dias da Silva, 165 3004-512 Coimbra, Portugal. Tel.: +351239790 596; fax: +351239403511.

E-mail addresses: cgsilva@estg.iplei.pt (C. Gomes da Silva), figueira@fe.uc.pt (J. Figueira), lisboa@fe.uc.pt (J. Lisboa), sbarman@ou.edu (S. Barman).
}

horizon for a given set of production resources and constraints. Such planning usually involves one product or a family of similar products with small differences so that considering the problem from an aggregated viewpoint is justified. The product demand data are assumed to be known with certainty; however, provisions for forecast error may be incorporated. The goal is to meet the forecasted product demand in a cost-effective manner. Typical costs related to APP include payroll, hiring/layoffs, overtime/undertime, and inventory shortage/backordering.

Numerous APP models with varying degrees of sophistication have been introduced in the last four decades. Holt 
et al. [23] developed one of the first APP models, commonly known as the LDR model. It was applied to a paint factory to generate a production plan using quadratic approximations to the actual operational costs of the firm. Silva et al. [2] extended the LDR model to develop a decision rule that considers a constant level of employment during the entire planning period. Hanssman and Hess [3] developed a model based on the linear programming approach using a linear cost structure of the decision variables. Haehling [4] extended the Hanssman and Hess [3] model for multiproduct, multistage production systems in which optimal disaggregation decisions can be made under capacity constraints. In addition, Bowman [5] introduced an APP model based on the linear programming technique using the transportation algorithm. Goodman [6] developed a goal programming (GP) model, which approximates the original nonlinear cost terms of the LDR model by linear terms and solves it using a variant of the simplex method. Rakes et al. [7] presented a chanceconstrained GP approach; a special case of stochastic programming to production scheduling that in corporates probabilistic product demand. A similar approach that uses a tabu search method has been proposed elsewhere [8]. Bowman [24] introduced the management coefficient model that generates a firm's aggregate production decisions by capturing and replicating its historical decisions with a multiple regression model. Vergin [9] developed a search-based simulation model and applied it to the paint factory problem [1]. Taubert [10] proposed the search decision rule (SDR) in order to generate an acceptable but not optimal solution to APP problems. Jones [11] formulated the parametric production planning (PPP) model with an objective function that describes the cost structure of a firm. In addition, Mellichamp and Love [12] proposed the production switching heuristics (PSH) model that is easy to implement and consistent with actual management practices. Ashayeri and Selen [13] applied an APP model to make strategic planning decisions for the pharmaceutical industry in The Netherlands.

There exist a few studies that integrate the interactive aspects of different functional areas of a company. Damon and Schram [14] analyzed the interrelationship between shortrun production rates and the roles of functional areas such as finance and marketing using an integrated model. Pega et al. [15] developed an integrated approach to address the aggregate planning problem and applied it to a firm, which yielded significant savings in the operational costs of the firm. In addition, excellent surveys of APP models can be found in Nam and Logendran [16], and Pan and Kleiner [17].

The approach presented in this study has to do with $m u l$ tiple criteria mathematical programming, which so far appears to have received less than sufficient research attention. Masud and Hwang [18] presented a multiple criteria linear programming model with four objectives, subject to a set of constraints. Another approach using a fuzzy linear programming was proposed by Wang and Fang [19]. Furthermore, a literature review indicates that the prior approaches on mul- tiple criteria mathematical modeling have one limitation in that they cannot generate a solution that optimizes all the important criteria simultaneously [20,21].

Generally, a gap exists between the theoretical contributions by researchers and the expectations of managers responsible for implementing aggregate production plans. The purpose of our research is to bridge this gap by introducing a multiple criteria mixed integer linear programming (MCMILP) model that is a realistic, practical alternative to more sophisticated, complex APP models. The practicability of the proposed model is demonstrated through its application in solving an APP problem of a Portuguese firm that produces construction materials. Furthermore, we developed a computerized decision support system (DSS) to aid managers for "solving" similar APP problems without having to familiarize themselves with the theoretical complexities of the model. The DSS, based on the learning-search-oriented concept, systematically searches for the best solution, if one exists. The operational features of the system are designed to generate, evaluate, and compare different solutions through a series of interactive steps [22]. The major advantage of the DSS is that it does not require the decision maker (DM) to comprehend the complexities of the multiple criteria algorithm to solve the problem. Rather, the DSS guides the DM "to solve" the problem in a way that requires the least amount of cognitive effort from the user.

The paper is organized as follows. Section 2 presents a description of the proposed MCMILP model. The main features of the interactive DSS are explained in Section 3. Section 4 includes an illustrative example of the DSS and discusses the results. Managerial implications of the research are provided in Section 5. Finally, conclusions and future research directions are presented in Section 6.

\section{The multiple criteria mixed integer linear programming model}

We developed the multiple criteria model considering three issues: company profit, client satisfaction, and the labor environment.

The decision variables are disaggregated by period and product families, or by period and worker type. The model also considers other production aspects such as the length of training periods, legal constraints on downsizing workers, production subcontracting and storage capacity.

\subsection{Notation}

1. Indices:

$m$

$q$

$n$ number of worker types number of product families number of periods (months) in the planning horizon 
2. Input parameters: $v_{i t}^{\mathrm{p}}$ $c_{i t}^{\text {lat }}$ $c_{j t}^{\mathrm{sal}}$ $c_{i t}^{\mathrm{inv}}$ $c_{j k t}^{\mathrm{red}}$ $c_{j t}^{\mathrm{tr}}$ $c_{j t}^{\mathrm{over}}$ $c_{i t}^{\mathrm{sub}}$ $c_{i t}^{\mathrm{p}}$ $h_{j i}$ $h_{j t}^{\mathrm{o}-\max }$ $h_{j t}^{\mathrm{r}}-\max$ $w_{j 0}$ $w_{j t}^{\max }$ $w^{\max }$ $p_{i 0}^{\text {over }}$ $p_{i t}^{\mathrm{dem}}$ $p_{i 0}^{\text {inv }}$ $p_{i t}^{\max }$ $p^{\max }$ $p_{i}^{\text {space }}$ $p_{i t}^{\mathrm{s}-\max }$ $p^{\text {space }}$ selling price per unit of product family $i$ in period $t$

late order delivery cost per unit of product family $i$ in period $t$

salary per worker for worker type $j$ in period $t$

inventory holding cost per unit of product family $i$ in period $t$

downsizing cost per worker for worker type $j$ hired in period $k$ and downsized in period $t$

hiring and training cost per worker for worker type $j$ in period $t$ overtime production cost per worker for worker type $j$ in period $t$ subcontracting production cost per unit of product family iin period $t$ production cost per unit of product family $i$ in period $t$

number of working hours needed per worker for worker type $j$ to produce one unit of product family $i$

maximum number of allowed overtime hours per worker for worker type $j$ in period $t$

maximum number of allowed regular hours per worker for worker type $j$ in period $t$

initial number of workers for worker type $j$

maximum number of workers allowed for worker type $j$ in period $t$ overall maximum number of workers allowed in each period (same for all the periods)

initial overdue level concerning product family $i$

demand level for product family $i$ in period $t$

initial inventory level for product family $i$

maximum number units of product family $i$ that can be produced in pe$\operatorname{riod} t$

overall maximum number of units to be produced (same for all the periods)

storage space needed per unit of product family $i$

maximum number of units of product family $i$ that can be subcontracted in period $t$

available storage capacity level $\mu$

$\alpha_{j}$

$\beta_{j}^{\text {reg }}$

$\beta_{j}^{\text {over }}$

$\xi_{j k}$

number of periods a worker must work before he/she could be downsized $^{1}$

required number of training periods per worker for worker type $j^{2}$

percentage of the regular working hours spent by each worker in training for worker type $j$

percentage of the overtime hours spentby each worker in training for worker type $j$

percentage of worker type $j$ hired in period $k$ that could be downsized (see footnote 1)

with $k=0, \ldots, t ; j=1, \ldots, m ; i=1, \ldots, q$; and $t=1, \ldots, n$.

3. Decision variables:

\begin{tabular}{|c|c|}
\hline$H_{j t}^{\mathrm{reg}}$ & $\begin{array}{l}\text { number of regular hours by worker } \\
\text { type } j \text { in period } t\end{array}$ \\
\hline$H_{j t}^{\text {over }}$ & $\begin{array}{l}\text { number of overtime hours by worker } \\
\text { type } j \text { in period } t\end{array}$ \\
\hline$W_{j t}$ & $\begin{array}{l}\text { overall number of type } j \text { workers em- } \\
\text { ployed in period } t\end{array}$ \\
\hline$W_{j t}^{\mathrm{tr}}$ & $\begin{array}{l}\text { number of type } j \text { workers in training } \\
\text { in period } t\end{array}$ \\
\hline$W_{j t}^{\text {hire }}$ & $\begin{array}{l}\text { number of type } j \text { workers hired in } \\
\text { period } t\end{array}$ \\
\hline$W_{j k t}^{\text {down }}$ & $\begin{array}{l}\text { number of type } j \text { workers hired in } \\
\text { period } k \text { and downsized in period } t\end{array}$ \\
\hline$P_{i t}^{\text {late }}$ & $\begin{array}{l}\text { units of late orders for product family } \\
i \text { in period } t\end{array}$ \\
\hline$P_{i t}$ & $\begin{array}{l}\text { units of product family } i \text { produced in } \\
\text { period } t\end{array}$ \\
\hline$P_{i t}^{\text {sto }}$ & $\begin{array}{l}\text { units of product family } i \text { stored in } \\
\text { period } t\end{array}$ \\
\hline$P_{i t}^{\text {sub }}$ & $\begin{array}{l}\text { units of product family } i \text { subcon- } \\
\text { tracted in period } t\end{array}$ \\
\hline
\end{tabular}

The variables $W_{j t}, W_{j t}^{\mathrm{tr}}, W_{j t}^{\text {hire }}$ and $W_{j k t}^{\text {down }}$ are restricted to integers.

\subsection{Performance criteria}

The model includes three performance criteria: profit maximization, late orders minimization and minimization of the changes in the work force level, as follows:

1. Profit maximization (this criterion maximizes the difference between the sales revenue and the sum of various

\footnotetext{
${ }^{1}$ Based on the Portuguese legislative requirements.

${ }^{2}$ Based on the industry average.
} 
cost components):

$$
\begin{aligned}
& \operatorname{Max} z_{1}=\underbrace{\sum_{i=1}^{q} \sum_{t=1}^{n} v_{i t}^{\mathrm{p}}\left(p_{i t}^{\mathrm{dem}}-P_{i t}^{\text {late }}+P_{i t-1}^{\text {late }}\right)}_{\text {sales revenue }} \\
& -\underbrace{\sum_{i=1}^{q} \sum_{t=1}^{n} c_{i t}^{\text {inv }} P_{i t}^{\text {sto }}}_{\text {storage costs }}-\underbrace{\sum_{i=1}^{q} \sum_{t=1}^{n} c_{i t}^{\text {late }} P_{i t}^{\text {late }}}_{\text {late deliveries costs }} \\
& -\underbrace{\sum_{j=1}^{m} \sum_{t=1}^{n} c_{j t}^{\mathrm{tr}} W_{j t}^{\mathrm{tr}}}_{\text {trainning costs }}-\underbrace{\sum_{j=1}^{m} \sum_{t=1}^{n} c_{j t}^{\text {over }} H_{j t}^{\text {over }}}_{\text {overtime costs }} \\
& -\underbrace{\sum_{i=1}^{q} \sum_{t=1}^{n} c_{i t}^{\mathrm{sub}} P_{i t}^{\mathrm{sub}}}_{\text {subcontracting costs }}-\underbrace{\sum_{j=1}^{m} \sum_{t=1}^{n} c_{j t}^{\mathrm{sal}} W_{j t}}_{\text {satary costs }} \\
& -\underbrace{\sum_{j=1}^{m} \sum_{k=0}^{n} \sum_{t=1}^{n} c_{j k t}^{\mathrm{red}} W_{j k t}^{\mathrm{down}}}_{\text {work force reducing costs }}-\underbrace{\sum_{i=1}^{q} \sum_{t=1}^{n} c_{i t}^{\mathrm{p}} P_{i t}}_{\text {production costs }} .
\end{aligned}
$$

2. Late orders minimization (this criterion minimizes the number of late orders during the planning period):

$$
\text { Min } z_{2}=\underbrace{\sum_{t=1}^{n} \sum_{i=1}^{q} P_{i t}^{\text {late }}}_{\text {late deliveries }} \text {. }
$$

3. Minimization of the changes in the work force level (this criterion minimizes the number of workers hired and downsized during the planning period):

$$
\text { Min } z_{3}=\underbrace{\sum_{t=1}^{n} \sum_{i=1}^{m} W_{i t}^{\text {hire }}}_{\text {hired workers }}+\underbrace{\sum_{j=1}^{m} \sum_{k=0}^{n} \sum_{t=1}^{n} W_{j k t}^{\text {down }}}_{\text {downsized workers }} .
$$

\subsection{Constraints}

The model includes three groups of constraints: workforce, production and inventory level.

- Work force: This group of constraints limits the number of workers employed globally and by worker type. They are required due to legal, technical, and/or operational constraints.

1. Definition of the number of workers for each type in each period:

$$
\begin{gathered}
W_{j t}=W_{j t-1}+W_{j t}^{\text {hire }}-\sum_{k=0}^{t} W_{j k t}^{\text {down }}, \\
j=1, \ldots, m ; \quad t=1, \ldots, n
\end{gathered}
$$

2. Legal limitations to downsizing workers:

$$
\begin{aligned}
& \sum_{\substack{w=k \\
1 \leqslant w \leqslant n}}^{k+\mu-1} W_{j k t}^{\text {down }}=0, \\
& \quad j=1, \ldots, m ; \quad k=0, \ldots, n .
\end{aligned}
$$

3. Firing constraints:

$$
\begin{aligned}
& \sum_{\substack{w=1 \\
w \geqslant k}}^{n} W_{j k w}^{\text {down }} \leqslant \xi_{j k} W_{j k}^{\text {hire }}, \\
& \quad j=1, \ldots, m ; \quad k=0, \ldots, n .
\end{aligned}
$$

4. Upper limit for each worker type:

$$
W_{j t} \leqslant w_{j t}^{\max }, \quad j=1, \ldots, m ; t=1, \ldots, n .
$$

5. Upper limit for the total number of workers:

$$
\sum_{j=1}^{m} W_{j t} \leqslant w^{\max }, \quad t=1, \ldots, n .
$$

6. Number of workers for each worker type currently engaged in training:

$$
\begin{gathered}
W_{j t}^{\mathrm{tr}}=\sum_{\substack{k=t-\alpha_{j}+1 \\
k \geqslant 1}}^{t} W_{j k}^{\text {hire }}-\sum_{\substack{w=t-\alpha_{j}+1 \\
w \geqslant 1}}^{t} \sum_{\substack{k=t-\alpha_{j}+1 \\
k \geqslant 1}}^{t} W_{j k w}^{\text {down }}, \\
j=1, \ldots, m ; \quad t=1, \ldots, n .
\end{gathered}
$$

7. Limitation of regular working hours:

$$
\begin{aligned}
& H_{j t}^{\mathrm{reg}} \leqslant h_{j t}^{\mathrm{r}-\max }\left(W_{j t}-W_{j t}^{\mathrm{tr}}\right)+\beta_{j}^{\mathrm{reg}} h_{j t}^{\mathrm{r}_{j} \max } W_{j t}^{\mathrm{tr}}, \\
& \quad j=1, \ldots, m ; \quad t=1, \ldots, n .
\end{aligned}
$$

8. Limitation of overtime hours:

$$
\begin{aligned}
& H_{j t}^{\mathrm{over}} \leqslant h_{j t}^{\mathrm{o}-\max }\left(W_{j t}-W_{j t}^{\mathrm{tr}}\right)+\beta_{j}^{\mathrm{over}} h_{j t}^{\mathrm{o} \_}{ }^{\max } W_{j t}^{\mathrm{tr}}, \\
& j=1, \ldots, m ; t=1, \ldots, n .
\end{aligned}
$$

Note that constraints 2 and 3 have to do with the Portuguese legislative requirements.

- Production: In this group of constraints we incorporate the limitation on the maximum production capacity, amount of subcontracting and production capacity by a worker, and the production balance requirement.

\section{Production balance:}

$$
\begin{aligned}
& P_{i t}+P_{i t}^{\mathrm{sub}}+P_{i t}^{\text {late }} \\
& =P_{i t}^{\mathrm{dem}}+P_{i t-1}^{\text {late }}+P_{i t}^{\text {sto }}-P_{i t-1}^{\text {sto }}, \\
& \quad i=1, \ldots, q ; \quad t=1, \ldots, n .
\end{aligned}
$$

10. Upper limit of the production capacity.

$$
\sum_{i=1}^{q} P_{i t} \leqslant p^{\max }, \quad t=1, \ldots, n .
$$


11. Upper limit of the production capacity by product:

$$
P_{i t} \leqslant p_{i t}^{\max }, \quad i=1, \ldots, q ; \quad t=1, \ldots, n .
$$

12. Upper limit on subcontracting:

$$
P_{i t}^{\mathrm{sub}} \leqslant p_{i t}^{\mathrm{s} \_\max }, \quad i=1, \ldots, q ; \quad t=1, \ldots, n .
$$

13. Production limitation for a worker in a period:

$$
\begin{aligned}
& \sum_{i=1}^{q} h_{j i} P_{i t}=H_{j t}^{\mathrm{reg}}+H_{j t}^{\mathrm{over}}, \\
& \quad j=1, \ldots, m ; \quad t=1, \ldots, n .
\end{aligned}
$$

- Inventory level: This set of restrictions assumes that the total storage capacity is limited for each period.

\section{Upper limit on storage capacity:}

$$
\sum_{i=1}^{q} p_{i}^{\text {space }} P_{i t}^{\text {sto }} \leqslant p^{\text {space }}, \quad t=1, \ldots, n .
$$

The solution to the model will include the following variables for each period in the planning horizon: the number of workers of each type to be hired, fired and trained, and the total number of workers required; the number of regular and overtime working hours for each worker type; and the amount of product to be produced, subcontracted, stored and backordered for each product family.

\section{An interactive decision support system}

We propose an interactive DSS based on the learning-search-oriented concept. The procedure includes two phases (see Appendix for details), the learning-oriented phase where the DM becomes familiar with different nondominated solutions, and the search-oriented phase where the DM learns more about the previous solutions and can be guided by the procedure to find the "best" solution, if it exists.

In the learning-oriented phase the DM searches for fixed reference points or evaluates the weights on each objective function without any guidance from the system. These reference points and/or weights are then used to obtain new solutions according to the Tchebycheff problem (for details see [20]). The DM can also reduce the admissible region by adding some constraints at the objective function levels. The aim of these constraints is both to preclude solutions that might be unacceptable and to enable a what-if analysis. Upon obtaining a solution, the DM has to decide whether the achievements at the objective function levels are acceptable or not. If they are, the solution is added to a generator set (GS), increasing the cardinality of the GS. The circumstances under which a given solution becomes acceptable do not necessarily represent a stopping condition.
This learning-oriented phase is repeated while the DM considers whether the information obtained is sufficient to build this preference structure. During this stage the DM could accept or reject any given solution.

The search-oriented phase begins when the DM decides to undertake a complete evaluation of the GS. The goals in this phase are: to provide the DM more information about the solutions retained from the previous phase; to help the DM understand the underlying trade-offs between the objectives; and to generate new potential solutions from the current GS.

Thus, the DM could choose from the following possible interactive options:

- Evaluating solutions: The DM chooses a solution from the GS he or she wants to evaluate. This happens when the DM is highly satisfied with some levels of the objective functions but not with the others. Consequently, the DM specifies the solutions that have satisfactory as well as unsatisfactory levels of objectives. For the former, the DM identifies the maximum amount to renounce in order to improve the level of at least one of the remaining criteria. Regarding the latter, the DM sets the minimum level of each objective that must be satisfied. These constraints are temporarily introduced to the original problem. If the solution appears to be satisfactory, it is then included in the GS, thus expanding the set.

- Neighborhood search: The DM selects a solution by using a set of weights and a reference point, and the system then searches some neighboring solutions. Of all the neighboring solutions, the ones that are found satisfactory by the DM are included in the GS. The DM may select more than one neighboring solution if he or she desires.

- Comparing solutions: The DM selects two solutions to compare. The choice of the solutions could be based on some apparently irrelevant aspects, when the achievement levels in the objective functions are somewhat similar. The DM could eliminate one of these solutions because it is indifferent from or inferior to the other.

- Removing solutions: The DM decides to directly remove a solution from the GS. Based on the knowledge accumulated during the interactive process, he or she considers that the solution is not good enough for implementation.

- Returning to the learning-oriented phase: The DM decides to return to the first phase (learning-oriented phase) because he or she wants to improve his or her knowledge about the nondominated region. This could result if the DS eliminates all of the solutions from the GS or is interested in finding new solutions not available in the current GS.

If the GS contains only one solution and the DM does not want to continue with any more interaction, then that would be the "best" compromise solution. Otherwise, using the unique element of GS, the DM may choose to find the "best" solution through the process of discovering, testing, and evaluating various alternatives. 


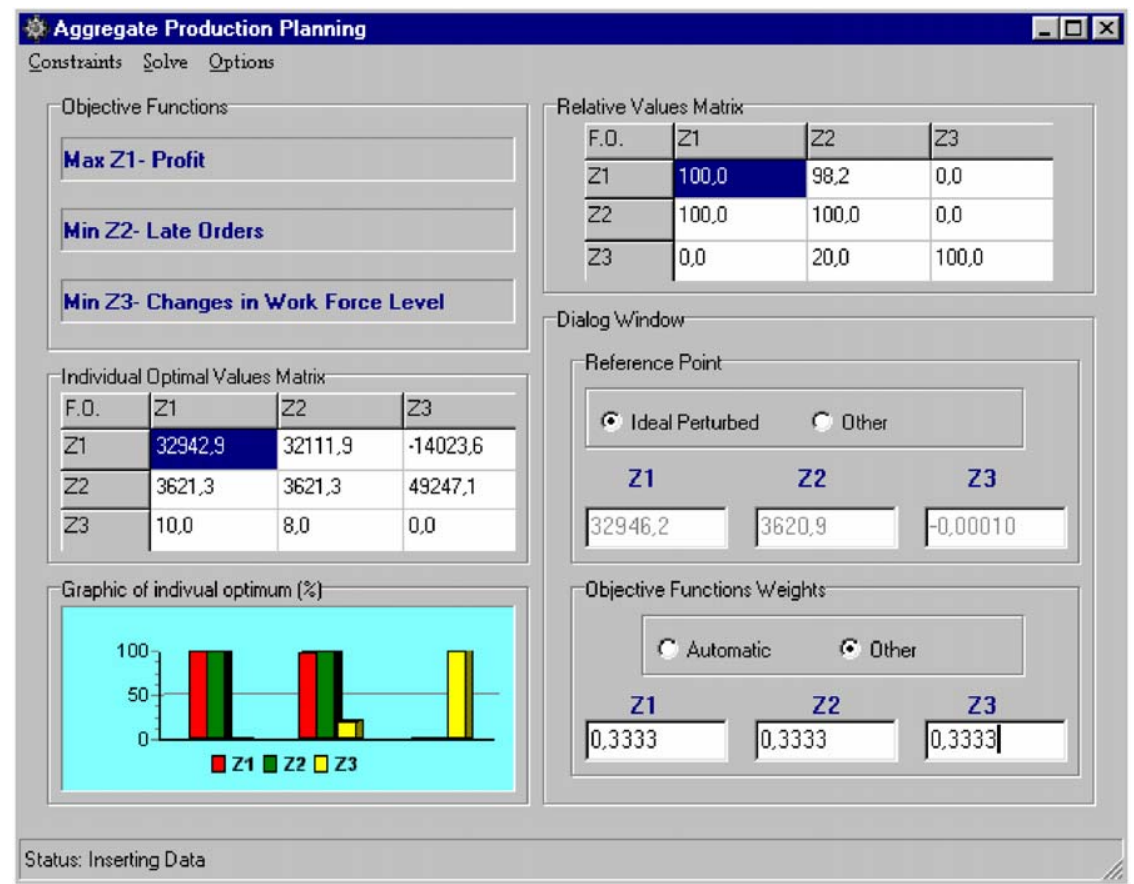

Fig. 1. Presentation menu.

We developed a DSS, written in Borland Delphi 4.0, to help the DM implement the MCMILP model. The DSS extensively uses graphs to represent and process information, enhancing the interaction with the DM. In addition, it is efficiently interfaced with EXCEL to retrieve the parameter values off the model and send back the planning maps and respective graphs containing the decision variable values. The structure of the DSS is presented in the appendix.

The following section illustrates the application of the model and the use of the DSS to solve an APP problem.

\section{An illustrative example}

In order to demonstrate the application of the model, we developed a case study using the data collected from the Portuguese firm. The firm makes polished granite for pavements and other products for the construction industry. The company has 36 workers with annual sales of nearly 500 million escudos (Portuguese currency). The products belong to three product families $(q=3)$ and there are three worker types $(m=3)$. For the firm, an adequate production plan for a 12month period $(n=12)$ is essential in order to cope with fierce competition as well as to provide low-cost products in a timely manner. We simulated an a posteriori decision making process with a fictitious DM. Note that the actual operational data could not be revealed for the sake of confidentiality.

Furthermore, with the three objective functions, the problem includes $n(8 m+3+3 q)+2 m$ constrains and $n(m(6+$ $n)+4 q$ ) decision variables, of which $m n 4+n$ ) are integers and $n(2 m+4 q)$ are continuous. Therefore, our illustrative example contains 792 decision variables (576 integers and 216 continuous) and 438 constraints. The specific numbers of constraints and variables for this problem have to do with the structure of the model and factors such as labor force restrictions, target inventory levels, production restrictions, etc. The DSS we presented to solve this problem is based on the Linear Programming solver LINGO 5.0 and was run on a COMPAQ computer with a Pentium $133 \mathrm{MHz}$ processor.

At the beginning of the interactive protocol, the DM only knows the optimal solutions for each criterion. This information provides the optimization limits for profits, late orders, levels of change in the regular work force, and the relative position of the remaining parameter values. As shown in Fig. 1, the matrix on the left includes the optimal value for each criterion (row), obtained by optimizing each objective function (column) separately. For example, the numbers $32111.9,3621.3$, and 8.0 in the second column represent the optimal amounts of profit (criterion 1), late orders (criterion 2 ) and changes in work force levels (criterion 3), respectively, when criterion 2 , the late orders, is optimized. The matrix on the right represents the relative values (or percentages) determined as follows: the value of the element $i \times j$ of this matrix is equal to

$\frac{100}{z_{i}^{*}-z_{i}^{n a d}}\left(z_{i j}-z_{i}^{n a d}\right)$,

where $z_{i}^{\text {nad }}$ corresponds to the worst possible value of row $i$, and $z_{i j}$ is the value of the element on row $i$ and column $j$ 


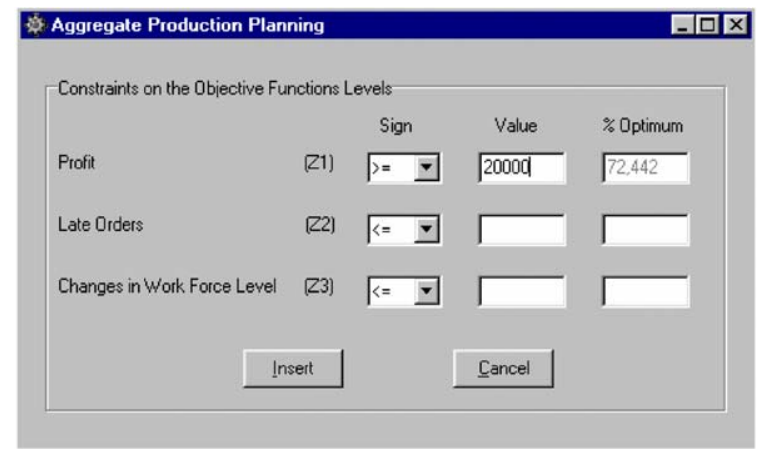

Fig. 2. Including restrictions.

of the left-hand side matrix. For example, 20, the value of element $3 \times 2$ of this matrix is obtained as follows:

$20=\frac{100}{0-10}(8-10)$.

At the bottom of the right-hand side of Fig. 1 is a dialog window that will allow the DM to define the reference points and the "weights" of the objective functions. As a result, the DM will be able to find a nondominated solution according to the preferred parameter values.

\subsection{First phase of the interactive method}

During the first phase, the DM searches for new solutions by introducing new constraints in the criterion space by assigning weights to each criterion and by specifying reference points. This is presented in the menu in Fig. 1. Two iterations of this type are presented below:

- First interaction. The information provided by the DSS at the beginning of the interactive protocol is the matrix of the individual optimal solutions and the corresponding percentage in the nondominated region. Unfamiliar with the mechanics of obtaining nondominated solutions, the DM accepted the default reference point. The defined vector of weights was $(0.333,0.333,0.333)$, and the problem is solved. The solution obtained is $S_{1}=(15663.0702,18428.7785,3)$, which corresponds to $(63.208 \%, 67.546 \%, 70 \%)$. The DM included this solution in the GS while reserving the option of choosing an alternative option later. We therefore have $\mathrm{GS}=\left\{S_{1}\right\}$.

- Second interaction. The second interaction has to do with the possibility of introducing constraints in the criteria space, which makes the interactive protocol easier. Next, a constraint was introduced to prevent profits from being less than 20 million escudos, equivalent to a requirement of $72.442 \%$ of the optimal profit. These requirements are introduced in the menu in Fig. 2, which follows from the Fig. 1 menu. The vector of the weights was fixed at the previous level $(0.333,0.333$, 0.333 ) so the weights did not "favor" any spacing from the reference point corresponding to the ideal perturbed

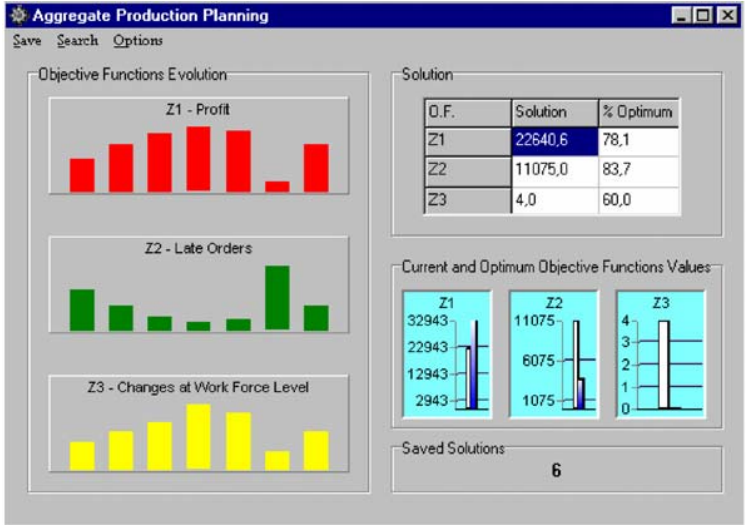

Fig. 3. First phase results.

point. The resolution of the problem leads to the solution $S_{2}=(22640.617,11074.970,4)$, which, in relative terms, corresponds to $(78.065 \%, 83.663 \%, 60 \%)$. This solution was added to GS, which is now composed of $\left\{S_{1}, S_{2}\right\}$.

Let us suppose that through similar iterations, the following four additional solutions were considered interesting by the DM: $S_{3}=(27547.777,6740.097,5), S_{4}=$ $(31390.459,3992.445,7), S_{5}=(29166.441 ; 5550.202 ; 6)$ and $S_{6}=(4710.002 ; 29741.965 ; 2)$. Thus, the GS is composed of $\left\{S_{1}, S_{2}, S_{3}, S_{4}, S_{5}, S_{6}\right\}$.

The evolution of the successive retained solutions during the decision making process is shown in Fig. 3. Let us assume that the DM considers the solutions included in GS are representative of the whole nondominated solution set and decides to use it as a generator set for new solutions. The DM now moves to the second phase with the search option.

\subsection{Second phase of the interactive method}

In this phase, the DM uses GS from phase one to carry out a more detailed search through the nondominated solutions space. The DM thus increases his/her knowledge about the solutions in the GS. In this phase, five interaction options are available: evaluating, comparing, removing and searching neighboring solutions, and returning to the learning-oriented phase.

At the beginning of the second phase, a menu is presented with all the retained solutions from the previous phase and the five interaction options.

Suppose the DM started by evaluating solution $S_{1}$ and considered the profit level unsatisfactory. Therefore, the minimum profit level was raised to 25 millions of escudos and the number of employees in the regular work force increased by one. Since the DM did not set the limit for late orders (Fig. 4), the problem therefore became impossible to solve. The DM consequently increased the number of employees by one more, which yielded solution $S_{3}$. Based on the results, the DM concluded that it would not be possible to obtain the desired profit level by changing the late orders 


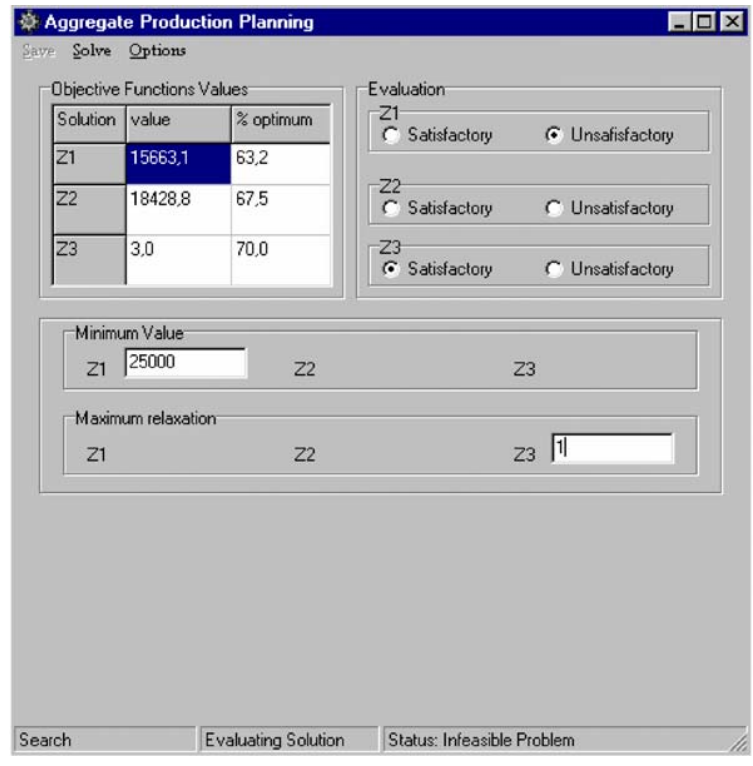

Fig. 4. Evaluation menu.

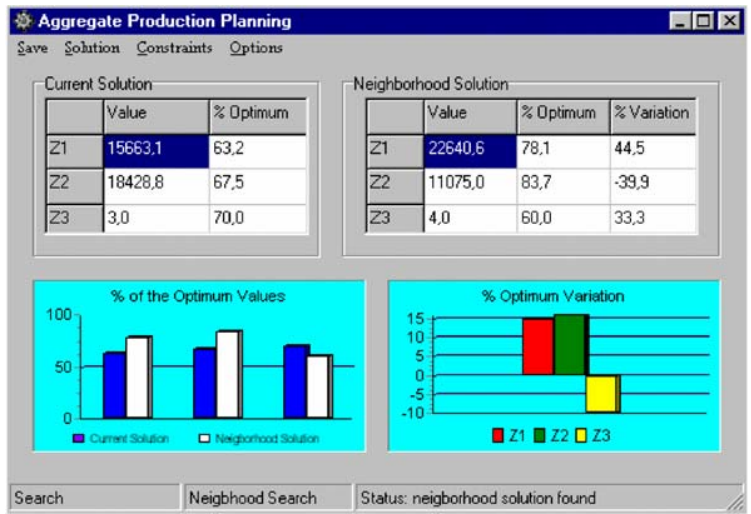

Fig. 5. Neighborhood solution search.

alone. The alteration in the number of employees in the work force was necessary to improve the profit level as well as to reduce the number of late orders.

The solution $S_{1}$ shows nearly the same percentage levels in the three criteria. The DM searched for a new solution in its neighborhood, and it was again $S_{2}$ (Fig. 5). The DM observed that $S_{6}$ has a very high value for the number of late orders and therefore excludes $S_{6}$ from the GS.

Upon comparing $S_{1}$ and $S_{2}$ (Fig. 6), the DM decided that the decrease in the work force performance criterion was acceptable because the profit level increased while the number of late orders decreased. The DM therefore eliminated $S_{1}$. We now have GS $=\left\{S_{2}, S_{3}, S_{4}, S_{5}\right\}$.

The DM now observes that solution $S_{4}$ is inferior to the other solutions with regard to the change in the work force level criterion. Since the GS contains solutions with a better result in this criterion along with comparable levels for the

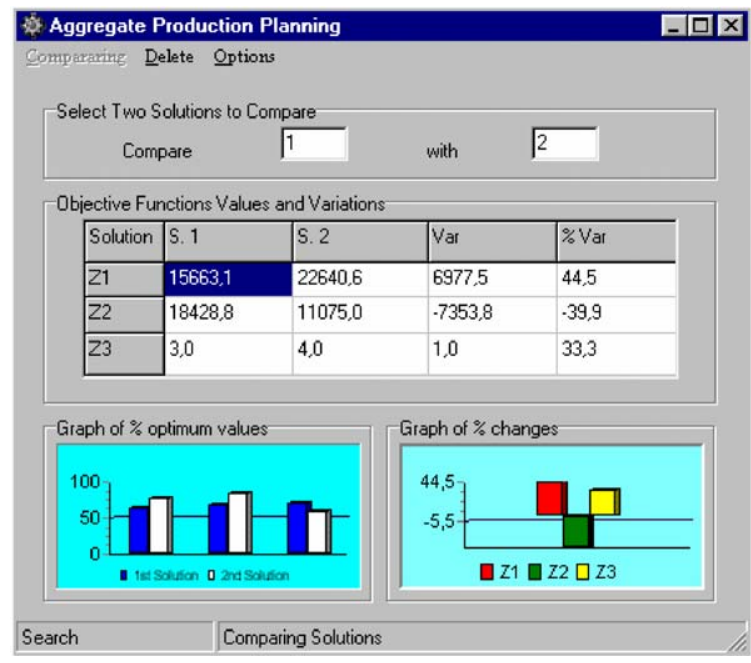

Fig. 6. Comparison menu.

others, the DM decides to drop $S_{4}$. The GS now includes only $S_{2}, S_{3}$, and $S_{5}$.

The DM next proceeds to eliminate $S_{2}$ from the GS because the volume of late orders is unsatisfactory since it exceeds $8000 \mathrm{~m}^{2}$. The current GS contains $S_{3}$ and $S_{5}$. The similarity of the change in the work force criterion for $S_{3}$ and $S_{5}$ necessitates a more detailed analysis of these two solutions. It appears that the increase in the profit level, along with the decrease in the number of late orders, outweighs the deterioration in the work force criterion. Therefore, $S_{3}$ is eliminated. The DM concludes that $S_{5}$ is the definitive solution of the interactive process.

Information on the decision variables for this solution is given in the menus in Fig. 7. It provides the DM with the necessary information to formulate the company's aggregate production plan for a 12-month horizon. Additional information may be obtained by analyzing the graphs in Fig. 8 .

Fig. 7 shows the results in which the sales and costs values are disaggregated by product family and by month, sales and production by month, and the sales and production accumulated in the planning horizon. It also shows the production level by product family and by month, late orders by month, and the overtime hours for each worker type by month.

\section{Managerial implications}

For practicing managers without the necessary mathematical knowledge or expertise on APP models, finding an analytical solution to the MCMILP model could be quite challenging. However, the computerized DSS presented herein makes the model an extremely useful problem-solving tool for such managers. The purpose of the DSS is to help managers, the intended users, obtain "the best solution" for a given problem without having to familiarize themselves with the mathematical complexities associated with the model or 

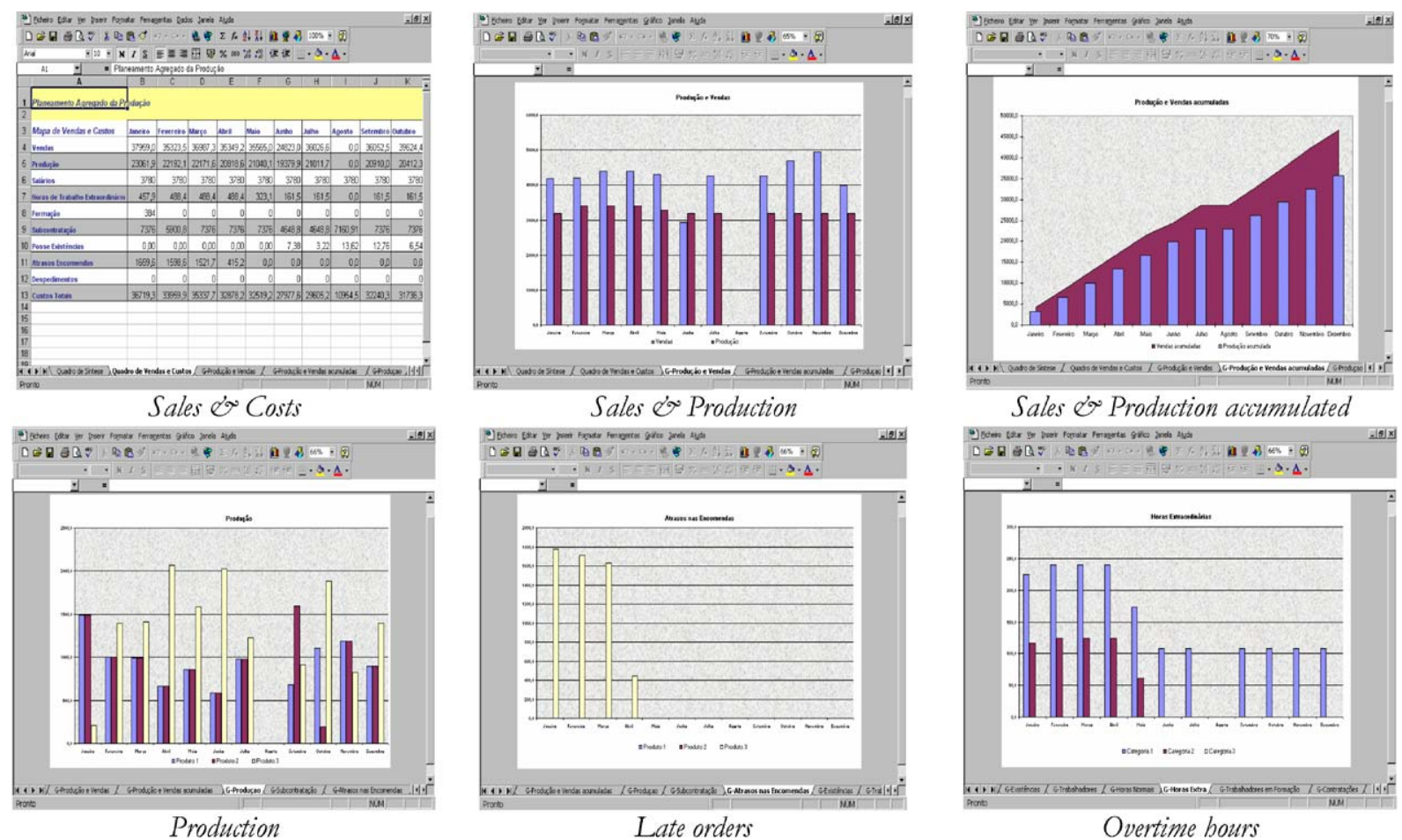

Sales \& Production accumulated

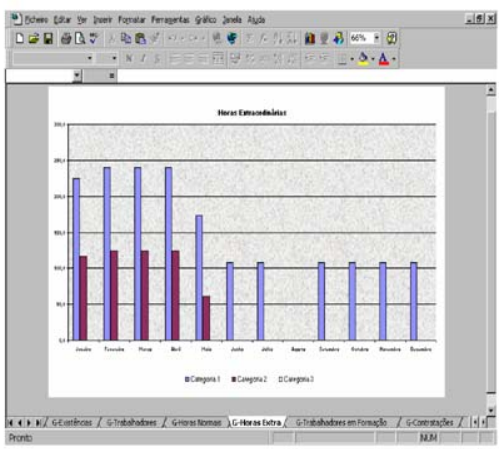

Overtime hours

Fig. 7. Excel graphs associated with $S_{5}$.

the solution method. The operational features of the DSS are quite user-friendly, involve a series of interactive steps to input the data, and use graphs and charts extensively to enhance the interaction between the system and the user.

The proposed DSS is based on the learning-searchoriented concept, which allows the user to generate, evaluate and compare various alternatives by explicitly considering different aspects of a typical aggregate planning problem. It is flexible enough in that various operational restrictions pertaining to different production systems can be easily incorporated into the model as constraints. Additionally, the built-in features of the DSS provide the manager the advantage of conducting the 'what-if' type of analyses to determine the extent to which the solutions are sensitive to the different parameter values of a given problem. Finally, in order to improve its usefulness, the system has been designed to have an efficient interface with EXCEL. Such an interface would enable the user to import input data (parameter values) directly from the organization's data base and/or to export the (textual/graphical) output of the model to the same or another data base for the purpose of storing or using as an input to another DSS.

\section{Conclusions and future research}

This paper presents a multiple criteria mixed integer linear programming model to solve aggregate production planning problems. The model has been developed to optimize three performance criteria for a set of workforce, production, and inventory-related constraints. The performance criteria include: profit, late orders, and the changes in the workforce level. In order to enhance its application in practice, a decision support system based on the model has also been included. We illustrated the use of the decision support system by applying the model to solve an actual aggregate planning problem faced by a Portuguese firm that produces construction products.

Over the past few decades, researchers have proposed many aggregate planning models with different levels of sophistication. However, many of these models have experienced limited industrial applications because of their methodological complexities, inflexible assumptions, and impracticable solutions. The research is an attempt to bridge this gap between the theory and practice of aggregate planning models. We believe that the DSS presented in this study will greatly enhance the application of the MCMILP model in practice, making it a better practical alternative to more sophisticated and complex aggregate planning models.

Future research might consider the development of an interactive DSS that will help managers cope with uncertain environments in the contexts of uncertainty and/or inaccuracy of forecast data. Another worthwhile avenue for extending this research would be finding ways to 
incorporate new constraints and objective functions in the model in flexible and efficient manners. Including modules to the current DSS that will allow the user to analyze the sensitivity of different operational and cost parameters is another possible logical extension to this research.

\section{Acknowledgements}

The second author would like to acknowledge the support from the Grant SFRH/BDP/6800/2001 and DIMACS research center for his sabbatical leave (August 2002-May 2003).

\section{Appendix}

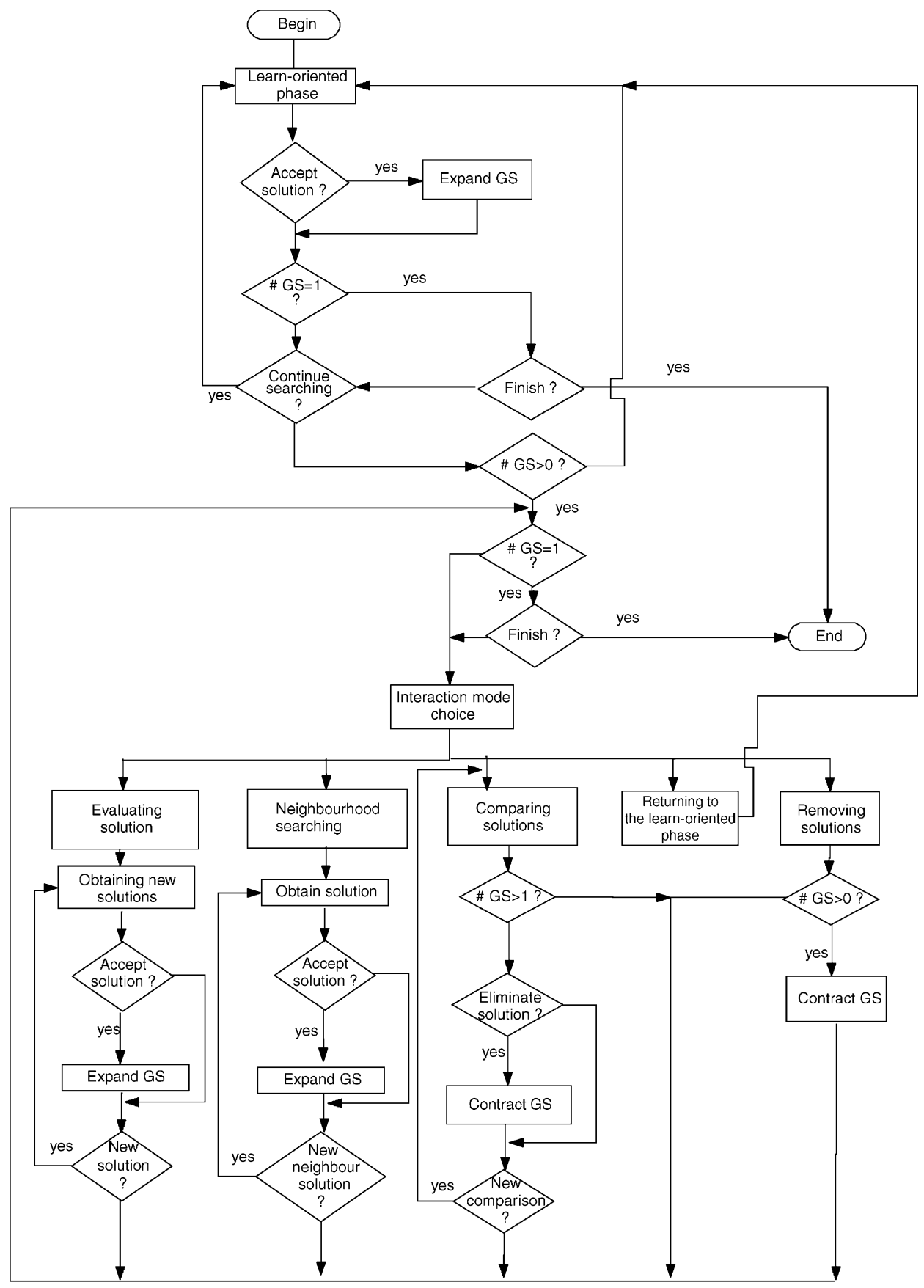

Structure of the interactive method 


\section{References}

[1] Holt CC, Modigliani F, Simmon HA. A linear decision rule for production and employment scheduling. Management Science 1955;2(1):1-30.

[2] Silva J, Lisboa J, Huang P. A labour-constrained model for aggregate production planning. International Journal of Production Research 2000;38(9):2143-52.

[3] Hanssman F, Hess S. A linear programming approach to production and employment scheduling. Management Technology 1960;1(1):46-51.

[4] Haehling LC. Production and employment scheduling in multi-stage production systems. Naval Research Logistics Quarterly 1970;17(2):193-8.

[5] Bowman EH. Production scheduling by the transportation method of linear programming. Operations Research 1956;4:100-3.

[6] Goodman DA. A goal programming approach to aggregate planning of production and work force. Management Science 1974;20:1569-75.

[7] Rakes R, Franz S, Wynne J. Aggregate production planning using chance-constrained goal programming. International Journal of Production Research 1984;22(4):673-84.

[8] Baykasoglu A. MOAPSS 1.0: aggregate production planning using the multiple-objective tabu search. International Journal of Production Research 2001;39(6):3685-703.

[9] Vergin RC. Production scheduling under seasonal demand. Journal of Industrial Engineering 1966;17:260-6.

[10] Taubert W. A search decision rule for the aggregate scheduling problem. Management Science 1968;14(6): B-343-59.

[11] Jones C. Parametric production planning. Management Science 1967;13(11):843-66.

[12] Mellichamp JM, Love RM. Production switching heuristics for the aggregate planning problem. Management Science 1978;24(12):1242-51.
[13] Ashayeri J, Selen W. A production planning model and a case study for the pharmaceutical industry in The Netherlands. Journal of Logistics: Research and Applications 2003;6(1/2):37-50.

[14] Damon W, Schram R. A simultaneous decision model for production marketing and finance. Management Science 1972;19(2):161-72.

[15] Pega MF, Lisboa JV, Yasin M. The financial aspects of aggregate production planning: an application of timeproven techniques. International Journal of Commerce and Management 2000;10(3\&4):35-42.

[16] Nam S, Logendran R. Aggregate production planning: a survey of models and methodologies. European Journal of Operations Research 1992;61:255-72.

[17] Pan L, Kleiner BH. Aggregate planning today. Work-Study 1995;44(3):4-7.

[18] Masud AS, Hwang CL. An aggregate planning model and application of three multiple objective decision methods. International Journal of Production Research 1980;18(6): 741-52.

[19] Wang RC, Fang HH. Aggregate production planning with multiple objectives in a fuzzy environment. European Journal of Operational Research 2001;133:521-36.

[20] Steuer R. Multiple criteria optimization: theory, computation and application. Chichester, UK: Wiley; 1986.

[21] Vincke P. Multicriteria decision aid. Chichester, UK: Wiley; 1992.

[22] Gomes da Silva C. Programação linear inteira mista multiobjectivo: aplicação ao planeamento agregado da produção. Master Dissertation, Faculdade de Economia da Universidade de Coimbra, 2000.

[23] Holt CC, Modigliani F, Muth JF. Derivation of a linear decision rule for production and employment. Management Science 1956;2(1):159-77.

[24] Bowman EH. Consistency and optimality in managerial decision making. Management Science 1963;9(2):310-21. 